Cahiers d'études italiennes

$1 \mid 2004$

NOVECENTO... E DINTORNI

Dire la guerre?

\title{
Tra Madrid e Guernica
}

Guerra civile spagnola e città ferite nella narrativa italiana (1996-2002)

Luciano Curreri

\section{CpenEdition}

Journals

Edizione digitale

URL: http://journals.openedition.org/cei/244

DOI: $10.4000 /$ cei.244

ISSN: 2260-779X

Editore

UGA Éditions/Université Grenoble Alpes

\section{Edizione cartacea}

Data di pubblicazione: 15 novembre 2004

Paginazione: 175-202

ISBN: 978-2-84310-057-4

ISSN: 1770-9571

Notizia bibliografica digitale

Luciano Curreri, «Tra Madrid e Guernica», Cahiers d'études italiennes [Online], 1 | 2004, online dal 15 mai 2006, consultato il 26 mars 2021. URL: http://journals.openedition.org/cei/244 ; DOI: https://doi.org/ $10.4000 /$ cei.244 


\title{
TRA MADRID E GUERNICA
}

\author{
GUERRA CIVILE SPAGNOLA E CITTÀ FERITE \\ NELLA NARRATIVA ITALIANA (1996-2002) ${ }^{1}$
}

\author{
Luciano Curreri \\ Université de Liège
}

1. Il titolo della comunicazione presentata, il 31 gennaio 2003, al seminario del CerCiC dedicato al tema della guerra, era " "Giravamo intorno a Madrid come di notte le farfalle intorno al lume". Aggiornamenti sulla narrativa italiana e la guerra civile spagnola (19962002) ». Titolare con citazione sciasciana era, innanzi tutto, omaggio a Claude Ambroise e a quei giovani amici e colleghi che ne seguivano il magistero (Silvia Roche e Christian Sorrentino). Ma era anche un primo modo per ancorare gli aggiornamenti, individuati tra vecchio e nuovo secolo e millennio, a una tradizione narrativa italiana che in rapporto alla guerra civile spagnola (1936-1939) pareva quasi inesitente e, nel migliore dei casi, perduta, trascurata dai lettori e dalla critica. In effetti, nell'anno intercorso, al di là degli elementi proposti nel seminario, la mia ricerca ha proceduto soprattutto in quella direzione, provando a colmare, à rebours, un vuoto di interesse e di dati. Due le tappe significative di questa promenade: un progetto di antologia per l'editore Einaudi, di Torino, con testi di Delfini, Jovine, Nitti, Sciascia, Lajolo, Lucarelli e Ramondino, purtroppo naufragato e ora in lettura presso altre case editrici torinesi (Aragno, S.E.I., Testo \& Immagine) ; un volume di critica, di prossima pubblicazione presso l'editore Bulzoni, di Roma, nella collana "Narrativa Novecento" diretta da Anna Dolfi, intitolato Le farfalle di Madrid. Narratori italiani e guerra civile spagnola. Come nella migliore tradizione, il seminario, l'incontro con amici e colleghi, attenti, curiosi, è stato l'input di una ricerca che si è poi oltremodo sviluppata, ben al di là di quella sede e della sua collocazione editoriale, i Cahiers d'études italiennes ai quali affido soltanto, per rispettare le consegne di Christophe Mileschi e Enzo Neppi, il discorso centrato sull'oggi e i percorsi tematici relativi alle città ferite ; sperando che tale discorso, alleggerito della prima parte e di parecchie note, conservi una sua autonomia e una certa validità, visto che non poggia propriamente sulla letteratura 'da biblioteca', quella incarnata da uno Sciascia, quella che tutti noi abbiamo promosso e forse troppo presto rimosso, e sposa una pratica quasi giornalistica, 'da libreria', 'da edicola' e 'da rete'. Ovviamente c'è un rischio, perché si gioca su ipotesi di rassegne e giudizi tranchants, e un vantaggio quasi immediato, perché si disegna una " mappa " per i naviganti. Per queste (e altre) ragioni il titolo è mutato e aggiunge a Madrid - che è, fin dall'inizio del conflitto e lungo l'intera durata dello stesso, simbolo di tutta la resistenza urbana ferita - l'" événement-symbole » di Guernica, il cui nome - " plus brûlant que les flammes de son incendie » (Pierre Vilar, Présentation a Herbert R. Soutworth, La destruction de Guernica. Journalisme diplomatie propagande et histoire, Paris, Ruedo ibérico, 1975, p. X-XI) - compare, più o meno significativamente, nei racconti e romanzi di Lucarelli, Carlotto, Ramondino. Inoltre, come si può desumere da certe note, che in modo contenuto aprono su altre realtà urbane, Madrid e Guernica rappresentano un più generale sviluppo della ricerca, dedicato alle Città ferite nella narrativa italiana del Novecento, anch'esso di prossima pubblicazione. Desidero infine ringraziare Enzo Neppi e Christophe Mileschi per l'invito e la simpatica accoglienza a Grenoble, in città e in quell'Università in cui ho potuto lavorare due anni come ATER, riuscendo a terminare e a discutere la mia tesi di dottorato. 


\section{Luciano Curreri}

A Paolo Scalabrin (Quartiere 1922-Russia 1943 [?]), scomparso in un'altra guerra, che forse era la stessa...

Qualcuno insinuò che stesse per scoppiare una bomba, come si era visto di recente in un film sulla rivoluzione russa.

- Dura da tanto tempo la guerra, nonna?

- No, figlia mia, quella di oggi è un'altra guerra. E altre ne verranno ancora.

Fabrizia Ramondino, Guerra di infanzia e di Spagna, Torino, Einaudi, 2001, p. 27 e 139-140.

\section{Oggi : limiti cronologici e ipotesi di rassegne tra Novecento e Duemila}

Oggi - l'oggi che è lo ieri più vicino, quello degli ultimi anni, a cavallo tra Novecento e Duemila - la narrativa italiana comincia ad appropriarsi, talora con una disinvoltura che può sembrare un po' eccessiva, della storia e della memoria relative alla guerra civile spagnola ; il cui cinquantenario è comunque già foriero, nella seconda metà degli anni Ottanta, di iniziative editoriali di taglio storico e politico, attestate, per esempio, da un volume di Angeli del 1987 curato da Natoli e Rapone ${ }^{2}$. Mentre Ettore Scola (1931), in quello stesso anno, nell'ampio sfondo storico de La famiglia, fa partecipare alla guerra civile spagnola il cugino Enrico (Giuseppe Cederna), già fuoriuscito a Parigi, che in quel conflitto trova pure la morte.

L'atteggiamento disinvolto di certi narratori sembra nascere in seno a una nuova attenzione, che è anche prodotta, nell'Italia di Alleanza Nazionale, da un ritorno più generale di riflessione sul fascismo, che si riaffaccia persino e con successo nella narrativa di consumo, nel noir e nel giallo soprattutto, da Carlo Lucarelli (1960) a Eraldo Baldini (1952), per esempio, da Carta bianca (1990) e L'estate torbida (1991) a Mal'aria (1998) ; e anche se per il primo la storia diventa, nel corso degli anni Novanta, un ingrediente di cui si può fare sempre più a meno, come vedremo, e se il secondo le preferisce il folclore, si tratta comunque di narrazioni che flirtano con lo scrupolo storico-politico, quale è anche quello

2. AA. VV., A cinquantanni dalla guerra di Spagna, a cura di Claudio Natoli e Leonardo Rapone, Milano, Angeli, 1987. 
che emerge, certo con più forza, proprio a metà degli anni Novanta, in Land and freedom (1995).

Ma il primo film storico di Ken Loach (1936), almeno in via formale (per veste e finanziamenti più robusti del solito), sembra funzionare meglio sul piano dell'attualità, al quale è legato per struttura narrativa (con la nipote che riattiva nel presente, contro quarant'anni di Franco, il significato della lotta antifascista del nonno). Mentre, su quello della storia, è apparso in parte retrodatato, specie per la tesi della causa rivoluzionaria tradita e persa in toto dai comunisti ortodossi ${ }^{3}$.

In Italia questo film ha avuto comunque una buona accoglienza e può essere stato, nel suo farsi tra passato e presente, storia e creazione, uno degli input del ritorno della guerra civile di Spagna. La scrittura di Ken Loach e di Jim Allen, lo sceneggiatore di Land and freedom, può aver spinto lo scaltro Lucarelli, e in prospettiva anche certo Cacucci (1955), a proseguire rapidamente parabole storico-romanzesche e biografie romanzate più o meno riuscite e vincenti : Guernica di Lucarelli è del 1996, a metà strada fra Tina (1991) e Ribelli (2001) di Cacucci, che tra l'altro passa in quegli anni - come Lucarelli, del resto - da piccole esperienze editoriali a grandi case editrici. E significativo, mi sembra, in relazione a certe superficiali dinamiche del mercato editoriale più che a implicazioni teoriche e culturali della cara e complessa postmodernità, è l'approdo di Cacucci a quella Feltrinelli che nel 1994 pubblica Sostiene Pereira di Antonio Tabucchi (1943).

Certo, nel romanzo di Tabucchi, sono in primo piano la Lisbona del 1938 e la dittatura di Salazar, ma la vicina guerra civile spagnola, il fascismo italiano e tant'altro costituiscono più di uno sfondo ${ }^{4}$. In partico-

3. Ma si veda quanto suggerisce invece Pino Cacucci, Ribelli!, Milano, Feltrinelli, «Serie Bianca », 2001, e poi " Universale Economica Feltrinelli », 2003, p. 11 : " (grazie, carissimo Ken Loach, che con Terra e libertà hai ridato voce internazionale ai ribelli schiacciati tra due immani dispensatori di falsità storiche : i franchisti e gli stalinisti)».

4. Di diverso parere è Nives Trentini che, in una peraltro ricca contestualizzazione di Sostiene Pereira, nota che " la Storia, pur documentata in tutta la sua drammaticità con l'assassinio di Monteiro Rossi, rimane uno sfondo ". Cfr. il recente Una scrittura in partita doppia. Tabucchi fra romanzo e racconto, Roma, Bulzoni, 2003, [p. 221-240], p. 234 ; ma già a p. 69 si legge : " la storia è solo uno sfondo e l'elemento strutturante del romanzo è piuttosto la formazione esistenziale del personaggio " (il discorso su Sostiene Pereira prosegue in tal senso, nonostante la studiosa riconosca « la presenza crescente della Storia », fino a p. 72). Nel saggio cercheremo brevemente di dimostrare, in rapporto ovviamente alla guerra civile spagnola, che Storia e formazione del personaggio sono strettamente legate. Qui in nota, invece, si vorrebbe aggiungere che forse, più che a leggere il romanzo, l'osservazione della Trentini, tesa ad evadere sostanzialmente una lettura politica "forzata " di Sostiene Pereira, può servire a 
lare, i riferimenti al conflitto spagnolo sembrano accompagnare la lunga, frammentaria e progressiva presa di coscienza di Pereira, un po' 'alla Bernanos' ; inizialmente non sono che accenni, frammenti, per l'appunto, dettagli incastonati, come la Lisbona e il Portogallo del 1938, in una monolitica e funerea deriva dell'Europa e del mondo negli anni Trenta ${ }^{5}$. Ma poi, tali riferimenti, si fanno via via autonomi e si impongono, in modo costante e progressivo, in una quindicina di luoghi del romanzo ${ }^{6}$.

interpretare l'omonimo film, che il regista Roberto Faenza ha tratto nel 1995 dal libro di Antonio Tabucchi, con la significativa collaborazione ai dialoghi dell'autore. In effetti, il lungometraggio non riesce sempre a riempire $\mathrm{i}$ « buchi ", $\mathrm{i}$ " vuoti » delle "storie zoppicanti » che il romanziere, secondo una sua stessa, suggestiva indicazione, fornisce " al cinema ", incontrando poi felicemente "registi che sono disponibili ai vuoti ». In Sostiene Pereira, mi sembra che certa " disponibilità ai vuoti » di Roberto Faenza si manifesti soprattutto nei confronti del contesto storico che, come quadro e senso di un'epoca ma anche come somma di eventi e esistenti di un plot, è rappresentato secondo gli stereotipi inflazionati di molto cinema engagé, diventando uno sfondo, per l'appunto, e spesso niente di più. Mentre il film riesce significativamente a catturare - nei dialoghi tabucchiani - che nel romanzo vengono assorbiti dall'universo del dottor Pereira e assimilano i continui e sempre più consistenti rilievi storici - la lunga e frammentaria presa di coscienza, l'apprendistato del « sostenere » del dottor Pereira (incarnato, con scelta felicissima, dal grande Marcello Mastroianni), e finanche la parabola esistenziale di altri due personaggi (nonostante l'interpretazione non esaltante di Stefano Dionisi, che è Monteiro Rossi, e di Nicoletta Braschi, che dovrebbe essere quella creatura di sogno, quella giovane femme fatale, quella silhouette inafferrabile, metamorfica che nel romanzo è Marta, "bellissima, chiara di carnagione, con gli occhi verdi [...] capelli castani che avevano riflessi rossi "; " capelli rossi [...] bella silhouette che si stagliava nel sole"; " Marta sembrava trasformata, quei capelli biondi e corti, con la frangetta e le virgole sulle orecchie, le davano un'aria sbarazzina e straniera, magari francese "). Per le citazioni in parentesi cfr. Antonio Tabucchi, Sostiene Pereira, Milano, Feltrinelli, 1994, p. 27-28, 96 e 99, 138 ; per quelle che precedono, invece, si veda, dello stesso Tabucchi, Come nasce una storia, in AA. VV., Scrittori a confronto. Incontri con Aldo Busi, Maria Corti, Claudio Magris, Giuliana Morandini, Roberto Pazzi, Edoardo Sanguineti, Francesca Sanvitale, Antonio Tabucchi, a cura di Anna Dolfi e Maria Carla Papini, Roma, Bulzoni, 1998, [p. 181-201], p. 196 (ma cfr. anche p. 186).

5. Cfr. Antonio Tabucchi, Sostiene Pereira cit., p. 14, 20-23, 28, 57 : «questa città puzza di morte, tutta l'Europa puzza di morte "; "Poi vide uno striscione [...] Onore a Francisco Franco. E sotto, in lettere più piccole : onore ai militari portoghesi in Spagna [...] era una festa salazarista [...] dalle loro corde malinconiche traevano una canzone franchista [...] non voglio andare in Italia, mi pare che la situazione sia ancora peggio della nostra [è Monteiro Rossi a parlare] "; " Oh, fece Pereira, la mia gioventù se n'è andata da un pezzo, quanto alla politica, a parte che non me ne interesso molto, non mi piacciono le persone fanatiche, $\mathrm{mi}$ pare che il mondo sia pieno di fanatici "; "viviamo in un mondo di teppisti, sono stati i teppisti [a parlare è il proprietario ebreo di una macelleria] ".

6. Ibidem, p. 37-38, 63-64, 80-81, 85-86, 97-98, 122, 128-129, 139-140, 145-147, 154156, 166-169, 177, 204. Stralciando, mi limito a esemplificare, in sequenza, dai primi due luoghi individuati nel romanzo : "Pericoloso, sostiene, l'articolo era pericoloso. Parlava della 
Più si procede nella lettura, insomma, più i riferimenti alla guerra civile spagnola diventano consistenti : da un rapido accenno, da un non comunque insignificante dettaglio narrativo, si passa a qualche pagina, fino a capitoli quasi interamente dedicati (o quanto meno ruotanti intorno) alla guerra, a quello che se ne sa, che se ne dice, che se ne scrive. Basta pensare, verso la fine del libro, al capitolo 19, centrato sul colloquio tra Pereira e padre Antonio, e al capitolo 20, dove assistiamo a quello col dottor Cardoso : capitoli e colloqui che non a caso sono essenziali per la presa di coscienza politica del dottor Pereira (e non solo per quella letteraria, che comunque può essere un valido supporto della prima e non slegata da essa) e per lo scioglimento del plot, con un uomo, un intellettuale, un traduttore, un giornalista che fa fronte a tutta la drammaticità, a tutta la violenza della Storia, reagendo con efficacia all'assassinio del giovane Monteiro Rossi, l'altro, l'amico, il figlio, il collaboratore letterario, il sovversivo, e colmando d'un tratto la distanza tra arte e vita, letteratura e engagement. Ché il dottor Pereira è engagé, è scrittore, giornalista engagé fin dall'inizio, in un certo senso, soprattutto se si presta giusta attenzione a quanto, risalendo a Sartre, suggerisce lo stesso Tabucchi in prospettiva anche extra-letteraria : " uno scrittore engagé è uno scrittore che si occupa dei fatti altrui ${ }^{7}$ ".

Il punto di vista di Pereira e il suo accesso alla responsabilità di fronte al mondo degli anni Trenta procede anche - e, si sarebbe tentati di dire,

profonda Spagna, della cattolicissima Spagna che García Lorca aveva preso come obiettivo per i suoi strali nella Casa di Bernarda Alba [...] non so se lei si rende conto, caro Monteiro Rossi, che in questo momento in Spagna c'è una guerra civile, che le autorità portoghesi la pensano come il generale Francisco Franco e che García Lorca era un sovversivo, questa è la parola : sovversivo »; « Pereira chiese a Silva cosa ne pensava di $[\ldots]$ quello che sta succedendo in Europa. Oh, non ti preoccupare, replicò Silva, qui non siamo in Europa, siamo in Portogallo [...] ma lo sai cosa sta succedendo in Germania e in Italia, sono fanatici, vogliono mettere il mondo a ferro e fuoco. Non ti preoccupare, rispose Silva, sono lontani. D'accordo, rispose Pereira, ma la Spagna non è lontana, è a due passi, e tu sai cosa succede in Spagna, è una carneficina, eppure c'era un governo costituzionale, tutto per colpa di un generale bigotto. Anche la Spagna è lontana, disse Silva, noi siamo in Portogallo. Sarà, disse Pereira, ma anche qui le cose non vanno bene, la polizia fa da padrona, ammazza la gente, ci sono perquisizioni, censure, questo è uno stato autoritario ».

7. A. Tabucchi, Come nasce una storia, in op. cit., p. 181. Per Sartre e una sua contestualizzazione in un panorama culturale francese il cui point de départ è Pascal cfr. Benoît Denis, Littérature et engagement. De Pascal à Sartre, Paris, Seuil, "Points Essais Série "Lettres”", 2000, volume da scorrere per intero, perché in esso Jean-Paul Sartre è presenza strutturante ; cfr. comunque, in particolare, nella prima parte, introduttiva, chiara ed efficace, il cap. III, L'écrivain engagé: une présence totale, alle p. 43-51, e nella terza ed ultima, il cap. XIII, L'apogée sartrien, alle p. 259-279. 
soprattutto - in base a quello che Pereira riesce a "sostenere » a proposito della guerra civile spagnola e dell'engagement che parte da Marta e dal cugino di Monteiro - Bruno Rossi, che " è in Portogallo per reclutare volontari portoghesi che vogliono far parte di una brigata internazionale ${ }^{8} "-$ e che investe lo stesso Monteiro e Pereira.

Del resto, è Tabucchi che ci invita a rinsaldare il personaggio, il protagonista con la Storia quando dice che "Pereira è infelice non solo per ragioni personali, per ragioni esistenziali $[\ldots]$ ma anche per la situazione storica che vive ${ }^{9} "$. Fare rientrare tale situazione storica, con i nazi-fascismi e un certo blocco totalitario europeo all'altezza del 1938, tra Portogallo e Spagna, Italia e Germania, nell'universo di un " vedovo, cardiopatico, grasso » personaggio, non significa necessariamente né farla restare uno sfondo, né proiettarla per intero nell'Italia elettorale del 1994, come vuole un po' Luca Doninelli, che in un articolo del 9 marzo di quell'anno, apparso sul "Giornale ${ }^{10}$ ", accusa Tabucchi di aver scritto " un libro di propaganda elettorale, ispirandosi a una visione ancora sovietica della Storia ».

Sostiene Pereira ha " un taglio politico indubbio, evidente » - lo dice lo stesso Tabucchi ${ }^{11}$ - ma questo taglio non riduce il romanzo storico, né lo

8. Cfr. Antonio Tabucchi, Sostiene Pereira cit., p. 80. Il cugino di Monteiro appare e scompare nell'intreccio, fra p. 80-81, 85-86, 97-98 etc., è presente anche in absentia, e dalla sua attività dipenderà, in un certo senso, la tragica soluzione della vicenda e la dolorosa presa di coscienza del vecchio gionalista, il dottor Pereira.

9. A. Tabucchi, Come nasce una storia, in op. cit., p. 192.

10. E di cui ridà notizia di recente «L'Indice", 2004, 4, p. 10, quasi a bilanciare la recensione che Luciana Stegagno Picchio fa dell'ultimo romanzo di Tabucchi, Tristano muore. Una vita, Milano, Feltrinelli, 2004, dove è ancora questione di Spagna, del Generalissimo (p. 62-65, per esempio) e di tant'altro Novecento : «Un Novecento che ha appena sceso il sipario sui nostri dubbi e certezze, ideologie e disillusioni. E che ora ci passa il testimone per il nuovo secolo. Ma chi testimonia per il testimone? ". Recensione appassionata, apocalittica e metafisica della "vecchia maestra " di Tabucchi, giocata sul refrain « di uno di quei vecchi e inutili, di quei Tristani quasi cadaveri che hanno capito come la storia sia un'illusione, un fantasma, anche se ormai non possono più farla, perché è già stata fatta " e chiusa poi su "un Antonio Tabucchi che vuol uscire dal Novecento senza voltarsi indietro ». E si citano questi passaggi della recensione al Tristano muore come esempio estremo di certa ricezione 'astorica' del romanzesco tabucchiano, certo favorita dall'autore stesso, a partire da Piazza d'Italia (1975), e opposta a quella iperattualizzante e diffusa soprattutto a partire dalla pubblicazione di Sostiene Pereira. E buon (e significativo) gioco hanno le "Vespe " del supplemento letterario de "Il Sole-24 ore », 2004, 114, p. 3, ad aggiungere : « Uscire ? Senza voltarsi ? Picchio del malaugurio ! Tocca ferro, Pereira ". Cfr. infine, anche per Spagna e guerra civile, A. Tabucchi, Piazza d'Italia. Favola popolare in tre tempi, un epilogo e un'appendice, Milano, Feltrinelli, 2003, p. 70, 91 e 132.

11. A. Tabucchi, Come nasce una storia, in op. cit., p. 193. 
fa diventare necessariamente neostorico, ovvero " più consono, per il "miscuglio di più fabule", ai modelli contemporanei in cui si fondono elementi storici "e allo stesso tempo gialli, rosa, d'azione, d'avventura, allegorici, politici, filosofici" 12 ". E l'escamotage del romanzo neostorico permette più che altro di apparentare Antonio Tabucchi o di provare ad avvicinarlo, con meno timore e più disinvoltura, tanto agli amati classici, Maupassant, Balzac e Tolstoj, citati in e per Sostiene Pereira insieme a Mauriac e Bernanos ${ }^{13}$, quanto, in rapporto alla guerra civile spagnola, a due 'giovani' come Pino Cacucci e Carlo Lucarelli e alla generazionalmente più vicina Fabrizia Ramondino (1936) ; autrice di un romanzo maiorchino che fa pensare a George Bernanos, citato nel testo e in un'appendice, e che si chiude con una nota di omaggi al romanzo ottocentesco in cui sono subito evocati Dumas e Dostojevskij, Balzac e Tolstoj ${ }^{14}$.

Del resto, ancor prima della pubblicazione di certi testi di Lucarelli, Cacucci e Ramondino, Carla Sodini e alcuni suoi allievi - nel chiudere una miscellanea, La guerra civile spagnola tra politica e letteratura, edita nel 1995 dalla Shakespeare and Company - pensano proprio a Sostiene Pereira e titolano pure, con mossa mimetica, Sostiene Tabucchi ${ }^{15}$, anche per una minima, sollecitata e attualizzante " partecipazione " d'autore raccolta in modo forzato nelle righe finali e tesa a enunciare tristi e certo avvertibili (e avvertite) analogie fra gli anni Trenta e il presente, con il ritorno dei nazionalismi, della xenofobia e del razzismo.

Tale intervento fonde così un richiamo storico-politico preciso con una sospetta o quanto meno dubbia e forzata attualizzazione, a scapito di una tenuta del testo letterario, del romanzo storico di Tabucchi, che è attualizzato e al tempo stesso fatto un po' scivolare all'indietro, perché chiamato, in un certo senso, a far le veci di una tradizione romanzesca italiana inesistente e/o perduta. Alla fine, le poche pagine dedicate a Sostiene Pereira quasi stonano in un volume corposo dove non c'è praticamente traccia della narrativa italiana ancorata in modo più o meno significativo al

12. Cfr. N. Trentini, Una scrittura in partita doppia. Tabucchi fra romanzo e racconto cit., p. 235, che utilizza " la ri-definizione del genere data da Margherita Ganeri (anche sulle tracce degli scritti di Ceserani) "; il rinvio è a Margherita Ganeri, Il ritorno postmoderno del romanzo storico : implicazioni teoriche e culturali, in "Allegoria ", 1997, 26, p. 112-120.

13. A. Tabucchi, Come nasce una storia, in op. cit., p. 195.

14. Fabrizia Ramondino, Guerra d'infanzia e di Spagna, Torino, Einaudi, 2001, p. 85, 416 e 425 .

15. Carla Sodini, A proposito della guerra civile spagnola : sostiene Tabucchi, in AA. VV., La guerra civile spagnola tra politica e letteratura, a cura di Gigliola Sacerdoti Mariani, Arturo Colombo, Antonio Pasinato, Firenze, Shakespeare and Company, 1995, p. 305-313. 
conflitto spagnolo (tra, potremmo suggerire, Delfini, Jovine, Sciascia, Lajolo, da un lato, e Vittorini, De Céspedes, Pavese, Brancati, Parise, Dessì, dall'altro) ${ }^{16}$; mentre campeggiano al solito la letteratura francese e l'angloamericana, la spagnola e finanche la tedesca, con Il grande esempio di Gustav Regler (1898-1963), scritto in tedesco nel 1937-1938 ma uscito in inglese nel settembre 1940, poche settimane prima del romanzo di Hemingway, da cui è subito oscurato ${ }^{17}$.

Lasciamo Tabucchi e proviamo a riapprodare a Pino Cacucci, per dire almeno che Tina è una biografia della Modotti (1896-1942), di un personaggio storico la cui vita è 'naturalmente' tesa al romanzo, come quella di Arthur Koestler (1905-1983), per esempio, che attraversa, in modo diverso, quasi gli stessi luoghi storici nella seconda metà degli anni Trenta. Tina, del resto, potrebbe rappresentare, per i nostri fini, una lenta ossimorica alba finesecolare, essendo pubblicata nel 1991, da Interno Giallo, e poi da Longanesi e Tea (fra 1994 e 1995 e con ristampe fino al maggio del 2001, se non mi sbaglio) : i capitoli sulla guerra civile spagnola sono sostanzialmente quattro, dal 23 al 26, con conclusione, se vogliamo, sul capitolo 27, e danno l'impressione di essere quattro racconti estrapolabili, tessere di un puzzle quasi a sé stante, che però partecipa all'operazione biografica, fra continuità e mistero ${ }^{18}$.

Il volume del 2001, invece, Ribelli! non opta per il monolite, per il personaggio unico, noto, d'eccezione, ma punta su tredici percorsi esi-

16. Cfr. la prima parte di questo lavoro, in Luciano Curreri, Le farfalle di Madrid cit.

17. Antonio Pasinato, Tragedia e speranza nella guerra civile spagnola. Il romanzo di Gustav Regler "Das große Beispiel " (1937-1938), in AA. VV., La guerra civile spagnola tra politica e letteratura cit., p. 231-249. Dico "finanche la tedesca " perché penso ancora, con Maurizio Serra, che gli italiani e i tedeschi che si scontrano, in patria e in Spagna, col fascismo e il nazismo, non riescano a offrire facilmente e nell'immediato un tributo alle belle lettere che traduca la realtà della guerra civile spagnola e quindi producano meno o un diverso tipo di opere. Cfr. Maurizio Serra, L'esteta armato. Il Poeta-Condottiero nell'Europa degli anni Trenta, Bologna, il Mulino, 1990, p. 130 ; ma lo stesso, a p. 131, ricorda, tra i tedeschi, Hermann Kesten (1900-1984) e il suo Die Kinder von Guernica (1939), con prefazione, non proprio compiacente, di Thomas Mann, che giudica non verosimile e eccessivo far parlare per 167 pagine un ragazzino di appena quindici anni, testimone della barbara distruzione di città e degli ultimi giorni della libertà e della giustizia. Altro, ovviamente, è il discorso relativo alla propaganda fascista e nazista. Per quella fascista, tra romanzo, teatro e cinema, rinvio ancora alla prima parte di questo lavoro, in Luciano Curreri, Le farfalle di Madrid cit.

18. P. Cacucci, Tina, Milano, Interno Giallo, 1991 ; Ivi, Longanesi, 1994, Tea 1995 e 2001, p. 140-169 e 170-174 ; cfr. poi, dello stesso, Ribelli cit., p. 43-57 e 59-63. 
stenziali dimenticati, che la ricordata Feltrinelli ha proposto nella «Serie Bianca " : una collana che sfuma un po' l'approdo narrativo cercato da Pino Cacucci e che vuole salvaguardare, quanto meno editorialmente, quella sensibilità e quella memoria un po' 'patinate' che permettono comunque di ripescare e applaudire - con una certa facilità - i ribelli : ovvero gli splendidi e solitari perdenti che hanno vinto in seno ai vari e tragici contesti storici che li hanno ospitati. E fra questi contesti riappare ancora la guerra di Spagna, con le vicende di Eulalio Ferrer - che dal Messico aspetta le scuse per i giovani antifascisti scampati al massacro in patria e lasciati marcire nei campi francesi - e di Quico, anarchico irriducibile e ironico, inventore di uno strano mortaio per sparare volantini antiregime sui cortei franchisti a Barcellona, la cui guerra continuerà fino al cinque gennaio del 1960 ; quel giorno, durante una delle sue operazioni antifranchiste, viene ucciso in un paesino spagnolo, che ricorda tanti altri piccoli e miseri villaggi della Spagna in guerra ed è quasi una sorta di Boadilla del Monte vent'anni dopo ${ }^{19}$.

Contestualizzare Cacucci fra monumenti letterari del passato più o meno noti, fra le testimonianze di un Koestler o di un Romilly (19181941), non significa evaderne la facile ricerca storica, o quella del mito a buon mercato, con i cattivi e i buoni in bella mostra sulla pagina-lavagna per un applauso adolescienziale che noi, certo, non possiamo regalargli.

Qui, ci si è un po' attardati su Cacucci per ragioni sostanzialmente cronologiche e si è passati da Tabucchi a Cacucci soltanto per provare a disegnare un orizzonte che fra romanzo e storia mira, comunque, al personaggio. La guerra, cioè, passa per una presa di coscienza di uomini e donne, di personaggi più o meno inventati, d'eccezione, e finanche consegnati a un certo riscatto eroico, abbracciato subito, o inseguito come lo scopo ultimo di una formazione, di una metamorfosi. La spettacolarizzazione dell'evento bellico, il richiamo altisonante e fortemente simbolico delle città ferite cede il primo piano a donne e uomini, al loro stare e agire nella storia, al loro sacrificio più o meno immediato. Come anche in Sciascia, nell'Antimonio (1960), dove però si alternano le due realtà, la città e l'uomo.

19. Il rinvio è a Esmond Romilly, Boadilla, London, Macdonald \& Co. (Publishers) Ltd, 1937. Cfr. la trad. it. di Mariapia Ghisalberti in Id., Boadilla. La mia guerra di Spagna, Introduzione di Hugh Thomas, Torino, Einaudi, 1974. "Boadilla del Monte è un piccolo e misero villaggio castigliano a circa venticinque chilometri da Madrid ", avverte subito Hugh Thomas nelle prime righe della sua Introduzione (p. VII-XIII) ; quel Hugh Thomas che è l'autore di The Spanish Civil War (1961), che resta un libro fondamentale e ancora oggi è la sintesi più completa sul conflitto spagnolo (pubblicato in italiano a Torino, da Einaudi, nel 1963, e in francese a Paris, da Laffont, nel 1961). 
Ricordiamo almeno, di sfuggita : « La città era intatta [...] ma fucilavano a non finire ". Frasi che sembrano essere la traduzione romanzesca di un appunto redatto da un funzionario di palazzo Chigi - forse Anfuso, per Renzo De Felice - che riferisce : "Si aggiunga che la Falange è quella che ha maggiormente "limpiado", ripulito, cioè, le città occupate fucilando il fucilabile ${ }^{20}$ ".

Insomma, i testi evocati di Pino Cacucci - traduttore, tra l'altro, del fortunato romanzo di Javier Cercas (1962), Soldados de Salamina, del 2001, che è diventato un film e che partecipa al ritorno narrativo della guerra civile spagnola nel mondo e in Italia, con la traduzione edita nel $2002^{21}$ - se presi nel loro insieme possono delimitare un decennio, compreso fra il 1991 e il 2001 ; un decennio appena trascorso, ancora in movimento, poco visibile e catturabile. Certo, Cacucci incarna - è giusto ripeterlo - un limite cronologico più che letterario, che ci può servire al di là dei due, tre input romanzeschi che il giovane narratore attiva sullo sfondo di una guerra che nella seconda metà di quel decennio si impone in altri approcci e in altre presenze autoriali del variegato panorama narrativo italiano.

Passiamo dunque in rassegna alcuni campioni esemplificativi, prima di offrire, degli stessi, una lettura centrata su Madrid, Guernica e le altre città ferite $^{22}$. E partiamo da Guernica, romanzo breve o racconto lungo di Carlo Lucarelli, pubblicato da Il Minotauro nel 1996 e riproposto da

20. Leonardo Sciascia, Gli zii di Sicilia, II ed., Torino, Einaudi, 1960, e poi Ivi, " Nuovi Coralli ", 1980, da cui si cita; da p. 181. Cfr. poi Renzo De Felice, Mussolini il duce. Lo stato totalitario. 1936-1940, Torino, Einaudi, 1981 e poi Ivi, "Tascabili », 1996, da cui si cita; da p. 378-379.

21. Javier Cercas, Soldados de Salamina, Barcelona, Tusquets Editores, 2001 e la trad. it. Soldati di Salamina, Parma, Guanda, 2002. Per il film cfr. Javier Cercas, David Trueba, Dialogos de Salamina. Un paseo por el cine y la literatura, Barcelona, Tusquets, 2003, e quanto ne diceva, in toni profetici e relativamente disposti, una volta tanto, a un certo compromesso, Goffredo Fofi, Le maschere dell'eroe, in "Film-TV ", 2002, 8, p. 114 : " "Soldati di Salamina" è pronto per farci un film astuto quanto il romanzo. Ma intanto, godetevi il romanzo! Sarà anche furbo, ma si legge d'un fiato ".

22. Per Madrid si scorra almeno Madrid, 1936-1939. Un peuple en résistance ou l'épopée ambiguë, dirigé par Carlos Serrano, Paris, Autrement, "Série Mémoires ", 1991 ; si vedano in particolare gli interventi dello stesso Serrano, Madrid 1936-1939 : un des repères de la conscience européénne, p. 12-18, e di Émile Temime, Le mythe et la réalité, p. 20-30, nella prima parte, che funge da Prologue, di José Carlos Mainer, Madridgrad ou le regard des autres, p. $102-$ 122, nella seconda, Un souffle épique, e di Julio Arostegui, L'agonie, p. 256-265, nella quarta parta, Du mythe à l'histoire; da leggere per intero la terza parte, La projection symbolique. Per Guernica cfr. H. R. Soutworth, La destruction de Guernica. Journalisme diplomatie propagande et histoire, cit. 
Einaudi nel 2000, annunciato come un capolavoro ma in realtà molto fragile e senza vero respiro storico, come sarà anche, ma ad altri livelli ed esiti, Lisola dell'angelo caduto (1999). In Guernica si sprecano e si giocano con facilità, in maniera scontata e superficiale, personaggi classici dell'epoca (la Modotti di Cacucci, Hemingway, Capa... finanche Lorca) e non si dichiarano gli input più recenti, magari non legati direttamente alla guerra di Spagna ma certamente alla propria tradizione e scuola, quella del noir, del polar più visionario. Pensiamo a un lungo racconto o romanzo breve di Pierre Magnan (1922), anch'esso titolato Guernica (1991), storia simbolica e orrorifica di sepolte vive confluita nella raccolta Les secrets de Laviolette, del 1992, ma pubblicata in Italia separatamente dalla Biblioteca del Vascello nel 1994 e riproposta, dopo il fallimento di quest'ultima, dalle Edizioni Robin nel 200123.

Facendo un breve passo indietro e ritornando al 2000, al 9 luglio del 2000 per la precisione, ecco poi comparire su "L’Unione Sarda » un racconto inedito di Massimo Carlotto (1956), Amore e odio di un gitano a Guernica; e il 23 agosto dello stesso anno, su "La Stampa ", una delle Storie di Vigàta e dintorni di Andrea Camilleri (1925), intitolata Uno strano scambio di persona, ci riporta ancora " al tempo della guerra di Spagna ${ }^{24}$ ".

Nel 2001 L'angelo della storia di Bruno Arpaia (1957) ${ }^{25}$ è dato alle stampe da Guanda, che nel 2002 ripropone, dello stesso autore, Tempo perso, il romanzo precedente, pubblicato nel 1997 da Tropea, centrato sulla rivolta-antefatto delle Asturie e collegato in parte per personaggi e sviluppo narrativo all'Angelo della storia, che approda alla guerra civile. Giocate su due tempi romanzeschi, il presente della ricerca, delle fonti e

23. Cfr. Carlo Lucarelli, Guernica, Milano, Il Minotauro, 1996 e poi Torino, Einaudi, "Tascabili-Stile Libero ", 2000 ; Pierre Magnan, Guernica (1991), in Les secrets de Laviolette, Paris, Denoël, 1992 e poi Paris, Gallimard, "Folio Policier », 1999, p. 77-152, e trad. it. di Mariella Aleggiani in Id., Guernica, Roma, Biblioteca del Vascello, 1994 e poi Ivi, Robin, 2001. E d'altro canto, e al di là dei giochi delle date per individuare possibili fonti di genere, anche il testo dell'autore italiano ha avuto una traduzione in francese, nata, mi sembra, da un interesse per il genere, il noir, e per i romanzieri italiani che lo frequentano, più che per il tema in sé, per la guerra civile spagnola e la città ferita del titolo. Anche se, è giusto ricordarlo, Guernica non viene pubblicata in quella "Série Noire " di Gallimard che aveva già ospitato, all'epoca, due titoli lucarelliani (Phalange armée, Le jour du loup) : cfr. C. Lucarelli, Guernica, traduit de l'italien par Arlette Lauterbach, Paris, Gallimard, 1998.

24. Questi ultimi due testi si possono leggere nei quotidiani sopra citati e nel sito degli stessi.

25. Bruno Arpaia, L'angelo della storia, Parma, Guanda, 2001 ; cfr. poi, dello stesso, Tempo perso, Milano, Tropea, 1997 e poi Parma, Guanda, 2002. 
dell'inventato testimone, e il passato della 'messa in scena', le pagine di Arpaia proiettano, alternano e incrociano, fra storia e invenzione, due uomini diversi, il critico e filosofo Walter Benjamin (1892-1940) e il testimone Laureano Mahojo, ovvero un intellettuale e un combattente repubblicano, un uomo d'azione ; quell' " uomo d'azione " che forse "è uno scrittore mancato ${ }^{26}$ " per Cercas e che invece Arpaia tende ad opporre, con risultati ambigui, al critico tedesco, giocando sul tandem a tratti un po' banalizzante di corpo e cervello, azione e studio.

Quasi a chiudere il decennio, Einaudi pubblica nell'ottobre del 2001 Guerra di infanzia e di Spagna della Ramondino, poderoso, importante romanzo, e anche al di là del dato storico e tematico che qui più ci interessa, la guerra civile spagnola. Notevoli, infatti, sono certi riinnesti autobiografici giocati sui " temi ricorrenti " della narrativa di Fabrizia Ramondino, "l'infanzia, l'emarginazione, l'esilio », sui fini e significativi recuperi delle prime Storie di patio (1983) e sulle oscillazioni curiose, sfrontate dei punti di vista infantili, di bambini e bambine a contatto con realtà geografiche lontane ma avvicinate dalla storia personale e collettiva, dispersa finanche, à rebours, tra Ottocento e Novecento, tra sfondi spagnoli e napoletani, con "vecchie case offese dalla guerra, dove s'ammassano famiglie venute da luoghi diversi ${ }^{27}$ " ; di bambine e bambini in rapporto favoloso con presenze quasi archetipiche come quella della nonna, che resta comunque creatura terrena, concreta, più della madre, e le cui caratteristiche ci ricordano un po' quelle che assume la figura, il personaggio della nonna nella prima parte di Ballata levantina (1961) di Fausta Cialente ${ }^{28}$.

Ma Guerra di infanzia e di Spagna della Ramondino è qui richiamata soprattutto per un capitolo, Storia del bambino di Guernica ${ }^{29}$, sul quale chiuderemo non casualmente il nostro discorso, approdando ancora a una città ferita il cui triste destino scuote l'immaginario infantile e maiorchino del romanzo e si sintonizza con il suo sviluppo in maniera simbolica e funzionale, fra alta resa letteraria e universale dell'offesa e leggi della struttura narrativa.

26. J. Cercas, Soldati di Salamina cit., p. 151.

27. Cito dalla Nota di quarta di copertina di Natalia Ginzburg a F. Ramondino, Storie di patio, Torino, Einaudi, 1983.

28. Fausta Cialente, Ballata levantina, Milano, Feltrinelli, 1961 ed ora, con Prefazione di Franco Cordelli, Il suo meraviglioso comunismo, e Postfazione di Paolo Terni, Ivi, Baldini \& Castoldi, 2003 ; la Parte prima, titolata, per l'appunto, La nonna, è alle p. 17-105.

29. F. Ramondino, Guerra d'infanzia e di Spagna cit., p. 199-208. 
Se invece vogliamo avventurarci nel 2002 e dunque estendere un po' l'arco cronologico di cui ci siamo serviti per la nostra prima ipotesi di rassegna, è facile accorgersi che la guerra civile spagnola continua a circolare nella nostra narrativa. Esplicitamente nel capitolo VII de La domenica di questa vita, primo romanzo dell'italianista Alberto Casadei (1963), pubblicato da Manni, di Lecce, nel giugno del 2002, dove - in una struttura simile a quella di Loach ma senza salto generazionale (non, insomma, nonnonipote ma padre-figlio) - si accenna a " un paesaggio imprecisato, cosparso di case distrutte dai bombardamenti dell'aviazione italiana o di quella tedesca » e a " terre martoriate, terrorizzate, semidistrutte ${ }^{30}$ ».

Implicitamente e problematicamente, pare poi che la guerra civile spagnola si insinui nell'ultimo libro di Alessandro Baricco (1958), Senza sangue, edito da Rizzoli nell'agosto 2002, dove i due tempi narrativi - separati da un cinquanta, sessant'anni, come ha suggerito Angelo Morino su "L'indice »- mettono insieme anche, rispettivamente, " un paesaggio travolto da anni e anni di guerra feroce " e Santander, "città del presente ", forse : " città spagnola della zona cantabrica, affacciata sul golfo di Biscaglia " che raccoglie, a guerra finita, a più di mezzo secolo di distanza, i segni non ancora scomparsi dell'orrore, con una casa di cura che sembra confusamente prendere il relais di un ospedale adibito a luogo di tortura durante gli anni di guerra ${ }^{31}$.

Va detto subito che il doppio e bipartito spazio-tempo narrativo (e lirico) di Baricco non conduce al romanzesco di Arpaia, in Italia, e soprattutto di Cercas, in Spagna, e non solo per la voluta, preziosa opacità ; un'opacità che certo lo allontana dal « racconto reale » verso il quale Cercas e il suo alter ego nel romanzo spingono con insistenza l'avventura umana di uno scrittore e di un falangista della prim'ora. Ad evadere le facili trappole dello schematismo ideologico pensa poi una battuta iterata con varianti nel testo e affidata a un felice, concreto personaggio femminile, la donna dell'alter ego. Una donna che non crede alle potenzialità romanzesche di una storia basata su un franchista e che esclama : "Se non fosse perché so che sei un intellettuale, direi che sei scemo. Non te l'ho detto fin dall'inizio che l'unica cosa da fare era scrivere di un comunista ? ${ }^{32}$ ".

30. Alberto Casadei, La domenica di questa vita, Lecce, Manni, 2002, p. 71 e 75. Come critico, Casadei si è occupato del tema della guerra, a cui ha dedicato due contributi : Id., $\mathrm{La}$ guerra, Roma-Bari, Laterza, "Alfabeto letterario ", 1999, e Romanzi di Finisterre. Narrazione della guerra e problemi del realismo, Roma, Carocci, 2000.

31. Angelo Morino, Una geografia fallace, in "L'Indice ", 2002, 12, p. 15, su, per l'appunto, Alessandro Baricco, Senza sangue, Milano, Rizzoli, 2002.

32. J. Cercas, Soldati di Salamina cit., p. 168. 
$\mathrm{Ma}$, al di là della boutade, è il titolo che, in gran parte, indica la strada giusta da percorrere per provare a capire e a raccontare, oggi, un evento come la guerra civile spagnola : Soldados de Salamina pensa infatti a un episodio di quell'avventura umana e storica " come se il fatto non fosse accaduto soltanto sessant'anni prima, ma si trattasse di un evento remoto quanto la battaglia di Salamina ${ }^{33}$ ".

I cinquanta, sessant'anni di Alessandro Baricco, dunque, seppur trasfigurati, perdono di significato di fronte a questa vastità, che ormai la memoria e la morale esigono, puntando non a una " geografia fallace » e a un'esibizione stilistica più o meno apprezzabile ; in virtù della quale " quanto è rappresentato è da ricercare al di là delle parole e delle immagini più immediatamente percebili, lì dove, malgrado le apparenze, tutto rimane per l'appunto immobile ${ }^{34} »$.

Proprio per il recente aprirsi (ed arricchirsi) del mercato italiano alla produzione romanzesca spagnola che attinge agli anni della guerra civile e ai suoi più o meno immediati dintorni, è bene anche porsi - rischiando ancora un giudizio e forse qualche ripetizione - in una prospettiva comparatistica che provi ad attivare rapide letture incrociate di testi finanche molto diversi, e non solo per esiti artistici e commerciali.

Dal più volte citato Soldados de Salamina, è bene ritornare, in tal senso, a ;Esa luz! (2000) di Carlos Saura (1932), per ricordare almeno come il noto regista - già implicato in altre evocazioni cinematografiche e non della guerra civile ${ }^{35}$ - scelga significativamente di esordire come roman-

33. Ivi, p. 40.

34. Cfr. ancora Angelo Morino, Una geografia fallace, cit.

35. Si vedano almeno le due presentazioni che ne fa, all'interno di un nutrito contesto, Marcel Oms, La guerre d'Espagne au cinéma, Préface de Pierre Broué, Paris, Cerf, 1986, p. 219-220, 288-291, che ha anche curato l'edizione dei Recuerdos de la guerra civil di Saura in M. Oms, Carlos Saura, Paris, Édilig, 1981. Faccio seguire solo alcune citazioni dalle p. 219220 e 289 de La guerre d'Espagne au cinéma, cit. : "Ce n'est qu'en 1973, avec La Cousine Angélique, que Carlos Saura montrera, du point de vue d'un vaincu, sa mémoire de la guerre, reconstituant les bombardements sur Barcelone de 1939 [...] et décrivant des comportements identifiables comme "franquistes " [...]. Avant La Cousine Angélique, Carlos Saura avait décrit métaphoriquement dans La Caza (la Chasse, 1965), les comportements des vainqueurs. Une séquence d'extermination de lapins en rase campagne renvoyait très explicitement à d'autres fusillades [...] Enfin, dans Le Jardin des délices (1970) un écran sur lequel étaient projetées des actualités de la guerre cédait la place à un groupe de gens en armes qui semblaient surgir du passé pour réveiller la mémoire assoupie du personnage central "; "Après la mort de Franco (1975), l'optique du cinéaste a changé et son projet aboutit finalement à cette espèce de revirement créateur extraordinaire qu'est Dulces horas (1982) où se 
ziere con una storia d'amore che principia, ancora, nel 1936, in Spagna, con la guerra che sconvolge l'esistenza di Diego, giornalista e scrittore già famoso a trent'anni, e quella di Teresa ; due vite raccontate tra Madrid, bombardamenti, provincia e fronte, tra corsivi atti a salvaguardare un certo punto di vista sul mondo, un'interiorità che può vacillare, e una narrazione più distesa, distaccata e onnisciente in caratteri normali ${ }^{36}$.

Un treno che sembra prendere anche Antonio Soler fin dall'inizio del suo El nombre que ahora digo (1999), storia dell'amore appassionato di Gustavo e Serena sullo sfondo vero, umano, perduto della guerra civile, di una Madrid morente, di una guerra lontana come quella di Cercas (e forse di Koestler) ma meno letteraria (e antica), di una guerra che non bisogna allontanare troppo e in modo artificiale e che bisogna tentare di riavvicinare al presente, all'oggi, a partire non da giornalisti e scrittori ma da personaggi marginali, da saltimbanchi, da innamorati della vita nella morte della guerra : "Ora so chi furono veramente quegli uomini che combatterono una guerra lontana [...] Ora so quali furono i desideri e i timori di quella gente, ma soprattutto conosco cosa si nascondeva dietro lo sguardo di Gustavo Sintora [...] Serena Vergara [...] Nessuno parlava e a nessuno importava di Gustavo Sintora né di come fosse giunto a Madrid. Non era altro che un filo di paglia che galleggiava nella marea impazzita della guerra [...] in fuga da Málaga. Mobili, catini, un pianoforte, animali, materassi, bambini e soldati che viaggiavano a passo d'uomo sui cassoni stracolmi dei camion. Bauli sventrati, sedie e morti per la strada ». Così principia l'odissea di Sintora, che " aveva perso la famiglia sotto un bombardamento » e che trova una nuova famiglia di " toreri e artisti [...] nani equilibristi ${ }^{37}$ " .

E dall'universo appassionato e funambolesco di Antonio Soler si può forse muovere verso Il tuo volto domani 1. Febbre e lancia (2003) di Javier Marias, docente universitario che nel suo decimo romanzo dà vita - un po' come Cercas, altro professore, all'Università di Gerona - a un «narratore, che è poi Marias stesso, sia pure sotto altri nomi e mansioni » ma che

mêlent $[\ldots]$ le vécu et sa représentation, le réel et sa mise en scène, où la chronologie ellemême paraît déréglée ". In questa prospettiva, si inseriscono altre opere di Carlos Saura e, per quel che ci riguarda, anche il romanzo a cui alludiamo rapidamente nel nostro discorso.

36. Cfr. Carlos Saura, ;Esa luz! (2000), trad. it. di Paola Tomasinelli in Id., Quella luce, Torino, Einaudi, 2002.

37. Cfr. Antonio Soler, El nombre que ahora digo (1999), trad. it di Paola Tomasinelli in Id., Il nome che ora dico, Milano, Marco Tropea, 2003, p. 7, 12-13 e 15-16. Cfr. la bella, entusiastica recensione di Paolo Collo, Amore e guerra nella Madrid morente, in "ttL-TuttoLibri ", supplemento culturale de "La Stampa », 2004, 1397, p. 3. 
con viva consapevolezza e partecipazione drammatica « rivive qualcosa che gli appartiene, vale a dire il clima della guerra civile spagnola ${ }^{38}$ ". E al di là dello stesso romanzo, che è stato accolto in modo entusiastico e compatto in Spagna e che ha invece un po' diviso la critica italiana ${ }^{39}$, è bene registrare quanto scrive Marias al momento della pubblicazione de Il tuo volto domani : "Quando sento dire spesso che è ormai ora di superare la faccenda della guerra, penso che la cosa non può succedere facilmente. Io, da bambino, ho sentito raccontare atrocità orribili, e mi rivolta che su questo si possa essere frivoli. E quando dico frivoli mi riferisco al modo sentimentale, blando, adulatore di cui è stata oggetto e continua a esserlo questa guerra oppure episodi accaduti. Ci sono cose che hanno bisogno di tempo per diventare materiale romanzesco più o meno palese. La guerra civile la stiamo ancora vivendo. La vita di mio padre, tanto per dire, è stata ridotta e limitata, perché aveva appartenuto alla parte repubblicana. E così è accaduto a tanti che hanno sofferto direttamente la guerra, prima, e poi il franchismo ${ }^{40} "$.

Vediamo insomma profilarsi un quadro composito ma in crescita, in cui immaginario romanzesco e mercato editoriale cercano e trovano - con una certa facilità (che non si traduce sempre in felicità inventiva e correttezza etica) - la memoria e la storia della guerra civile di Spagna.

Insieme, ancora, a diari, testimonianze, a studi storici ${ }^{41}$, che qui possiamo evocare rapidamente - e sempre con intenti esemplificativi - in relazione agli aggiornamenti romanzeschi, per valorizzare in tal senso il

38. Si cita da Angela Bianchini, Marias a Oxford. Echi di guerra civile, in " ttL-TuttoLibri », supplemento culturale de "La Stampa », 2003, 1382, p. 3.

39. Per esemplificare rapidamente la reazione della stampa mi servo dell'appena citata e favorevole Angela Bianchini, da un lato, e dall'altro della recensione assai dura di Danilo Manera, Un'orgia di parole, in "L'Indice », 2003, 3, p. 17.

40. Cfr. ancora A. Bianchini Marias a Oxford. Echi di guerra civile cit.

41. Giusto rinviare, almeno in nota, a qualche titolo che tra Novecento e Duemila, tra Francia e Italia, tra inediti e riproposte, ha richiamato significativamente l'attenzione dei lettori e degli specialisti sulla guerra civile spagnola e sulla tragica scomparsa della democrazia in Europa e nel mondo. Cfr. per esempio Les Espagnols et la guerre civile, Textes rassemblés et présentés par Michel Papy, Biarritz, Atlantica, 1999 ; Jean-François Berdah, La démocratie assassinée. La République espagnole et les grandes puissances. 1931-1939, Paris, Berg, 2000 ; Gabriel Jackson, La Repubblica spagnola e la guerra civile. 1931-1939, Milano, Net, 2003 (ma già Ivi, Il Saggiatore, 1967, trad. it. di The Spanish Republic and the Civil War. 1931-1939, Princeton, Princeton University Press, 1965) ; Gabriele Ranzato, L'eclissi della democrazia. La guerra civile spagnola e le sue origini, Torino, Bollati Boringhieri, 2004. 
ritorno o la comparsa di alcuni di questi volumi sul mercato : ritorno o comparsa funzionali al panorama narrativo di fine e inizio secolo che ci interessa e che possiamo infine situare - restringendo e avvicinando il campo d'indagine - fra il 1996 e il 2002, anno davvero propizio anche per i diari e gli studi.

Fra coloro che trasferiscono in Spagna ${ }^{42}$ il travaglio maturato nello scontro col fascismo in Italia citerei almeno Aldo Morandi (1896-1975) e il suo In nome della libertà. Diario della guerra di Spagna 1936-1939, edito, nel 2002 per l'appunto, da Mursia, a cura di Pietro Ramella, e soprattutto Camillo Berneri (1897-1937) e il suo Mussolini alla conquista delle Baleari, uscito ben tre volte, fra il 1937 e il 1938, in spagnolo e in francese, ed oggi - a partire dal febbraio del 2002 - disponibile in italiano grazie all'iniziativa dell'editore Giuseppe Galzerano di Casalvelino Scalo (Salerno), che presenta questo studio insieme a Claudio Venza, professore di Storia della Spagna contemporanea a Trieste, già curatore di un volume importante come Le passioni dell'ideologia. Cultura e società nella Spagna degli anni Trenta (1989) ${ }^{43}$.

Lo studio di Camillo Berneri può essere di una certa utilità per leggere Guerra di infanzia e di Spagna di Fabrizia Ramondino, anche al di là delle Brevi notizie sulla guerra civile spagnola a Maiorca riportate in Appendice al romanzo e tratte dal libro di Josep Massot i Muntaner, La guerra civil a Mallorca (1976), e soprattutto al di là dell'antecedente di culto sulle Baleari, Les grands cimetières sous la lune (1938) di Georges Bernanos, che comunque è citato dalla Ramondino nel settimo capitolo della prima parte del suo romanzo ${ }^{44}$.

42. Cfr. ancora Maurizio Serra, L'esteta armato. Il Poeta-Condottiero nell'Europa degli anni Trenta cit., p. 121-146; in particolare p. 130.

43. Si vedano rispettivamente Aldo Morandi, In nome della libertà. Diario della guerra di Spagna 1936-1939, a cura di Pietro Ramella, Milano, Mursia, 2002 ; Camillo Berneri, Mussolini alla conquista delle Baleari, Casalvelino Scalo - Salerno, Galzerano, 2002 ; Le passioni dell'ideologia. Cultura e società nella Spagna degli anni Trenta, a cura di Claudio Venza e altri, Trieste, Editre, 1989. E di Giuseppe Galzerano è giusto segnalare anche, come autore oltre che editore, il volume dedicato a Vincenzo Perrone. Vita e lotte, esilio e morte dell'anarchico salernitano volontario della libertà in Spagna, Casalvelino Scalo - Salerno, Galzerano, 1999.

44. Cfr. ancora Fabrizia Ramondino, Guerra d'infanzia e di Spagna cit., p. 415-417 e 85. Il lungo pamphlet di Bernanos, in Francia, è costantemente ristampato e si può leggere in edizione economica, con testo integrale, nei " Points » Seuil ; in Italia, l'ultima edizione, se non mi sbaglio, è quella di Milano, Il Saggiatore, «L’Arco », 1996. 


\section{Romanzi, racconti e percorsi tematici : Madrid, Guernica e le altre città ferite}

Iniziamo dunque a leggere uno per uno i romanzi e racconti citati, seguendo l'ordine cronologico ma cercando ormai di reperire i percorsi tematici centrati, per l'appunto, su Madrid, Guernica e le altre città ferite ; percorsi in virtù dei quali l'insieme individuato nelle ipotesi di rassegne sembra potere - pur nella diversità evidente dei suoi componenti - coagularsi e resistere al di là del singolo testo e al di là dello stesso giudizio critico.

In questa prospettiva d'indagine, Guernica di Carlo Lucarelli, anche se è un testo che non ci convince, può comunque essere un utile punto di partenza per seguire la parabola della città bombardata - il 26 aprile 1937 - nella narrativa italiana degli ultimi anni. Di più. Nel legare l'iter romanzesco a un percorso urbano che in quella città ferita trova il suo approdo - da Teruel a Madrid, passando per Sigüenza, e da Madrid a quel 'contenitore' che è Guernica ${ }^{45}$ - Lucarelli offre una mappa, dove è anche, in posizione centrale, quella capitale che è simbolo di tutte le città spagnole ferite dalla guerra, come si è detto. Il Prologo stesso, del resto, la pone subito in evidenza, associandole una data, il 10 aprile 1937, che rende significativamente vicino il triste futuro della città basca, già rappresentabile a partire da Madrid. La notte offre infatti alla capitale un "cielo buio " perché « forse anche le stelle erano venute giù con le bombe degli stukas, il giorno prima ". Nella stessa pagina segue non a caso l'evocazione della Legione Condor. E subito dopo, a Teruel, il protagonista, il doppiogiochista Filippo Stella, "ubriaco ", si trova a vivere una notte quasi uguale, " tra tuoni di contraerea e fulmini di traccianti ${ }^{46}$ ". Arruolato di forza nel CTV - Corpo Truppe Volontarie - Stella deve aiutare, in qualità d'attendente, il capitano Degl'Innocenti a ritrovare « la salma di un suo amico compagno di corso, colpito al petto dalla mitraglia durante l'assalto di Guadalajara ", il tenente e nobile Vittorio Emanuelli. Costui, si scoprirà via via, non è affatto morto e si è trasformato in una specie di mostro, di lupo mannaro che a grandi passi muove verso l'orrore di Guernica. Qualche luna piena, qualche teschio, qualche urlo o ululato e il gioco è fatto. La guerra civile serve un genere, che passa in rassegna se stesso in un

45. Cfr. ancora H.R. Soutworth, La destruction de Guernica. Journalisme diplomatie propagande et histoire cit.

46. Per questa citazione e le seguenti cfr. C. Lucarelli, Guernica cit., p. 5 e 13, 17, 86 e 93, 104, 65 e71, 73-74, 40. 
altro orizzonte epocale, nel quale il destino della città ferita, di Guernica, funge da catalizzatore di tutto il male della guerra : « tra i fascisti si dice che raderanno al suolo il paese con uno dei bombardamenti più duri che si siano mai visti. Sarà come se la guerra scendesse dal cielo su Guernica ». Ma anche di tutto il male dell'umanità trasfigurata, mostruosa che vi prende parte : «Adesso [Emanuelli] vaga per la Spagna e che sia un disertore, un assassino impazzito o l'anima stessa della guerra, sembra stia puntando su Guernica ». Alla fine, prima ancora di arrivare a Guernica, o forse proprio a Guernica, in un punto ormai imprecisato della Spagna, del mondo ferito, e in un'attesa diluita e apocalittica, i due soldati, il capitano e l'attendente, assomigliano non a caso agli eroi di Cervantes, Don Chisciotte e Sancho Panza : " uno alto, magro e sul cavallo, a delirare con le braccia aperte e una bacinella da barbiere sulla testa e l'altro basso, piccolino e grasso, che lo seguiva sopra un mulo ". Ma prima di questo approdo tragicomico, frutto di un'inchiesta da polar visionario, un po' alla Magnan, ci sono altre pagine da appuntare, abbastanza interessanti, anche se non tutte in relazione al tema delle città ferite, dalle quali comunque conviene cominciare, magari a partire da quei " gironi infernali " che « indicano la strada per Madrid »: «Da uno strappo del telone io guardavo fuori nella terra di nessuno [...] C'erano case sventrate e senza tetto, dalle travi irte sulle mura come capelli dritti di paura e buchi alle porte e alle finestre, come bocche e occhi spalancati "; "Ero in una chiesa, una piccola cappella di campagna, distrutta dal fuoco che l'aveva ripiegata su se stessa ». E poi Madrid, tra periferia e centro, con i suoi abitanti e le sue ronde : " Era quasi sera quando arrivammo alle porte di Madrid, buia per l'oscuramento, nera e frastagliata di macerie contro la penombra arrossata del tramonto. In periferia le case non c'erano più e la gente stava tutta fuori nonostante il coprifuoco, in mezzo alla strada, lontano dai muri pericolanti delle facciate vuote $[. .$.$] gli feci vedere che lì tutti camminavano$ un po' curvi e più lenti, come se ce le avessero sempre sulle spalle un po' di quelle macerie di case [...]. In città le case erano meglio e la gente stava dentro, dietro le pezze, i sacchi e i fogli di giornale che fasciavano le finestre [...] trattenevamo il respiro, schiacciati contro il muro umido di un androne e a Puerta del Sol, dove una bomba aveva scavato un cratere, evitammo per un soffio una ronda comunista ». In altre pagine, più facili ma riuscite, si passa invece dalla presenza di Pinocchio - il " giocattolo italiano » che compare anche nel romanzo della Ramondino - al disoccupato che prima di fare il legionario in Spagna fa la comparsa del legionario in Scipione l'Africano (1937) e che preferisce, ovviamente, la finzione alla realtà : "Lì per lì la paga è buona [...] e mi arruolo [...]. Ma quando 
sbarco a Malaga e mi trovo gli ufficiali di Franco che ci salutano urlando Viva la muerte... in te cul viva la muerte! Io non ci sto... così mi sbattono in guardina ". E così Viva la muerte non diventa la "versione iberica del nostro "Me ne frego !"”, come un editoriale di " Prospettive ", la rivista diretta da Curzio Malaparte, voleva farci credere a guerra finita, in " una sorta di romanticismo strapaesano ", secondo Aldo Garosci ${ }^{47}$.

Per passare al racconto di Massimo Carlotto - che per presenze gitane e altri spunti è possibile avvicinare al romanzesco, certo più corposo, di Soler - si appunterà subito che la sua " forme brève " - ancora più raccolta di quella di Lucarelli - non lascia strutturalmente spazio a descrizioni particolareggiate, che possano magari procedere, in un iter urbano come quello appena evocato, oltre la città del titolo, Amore e odio di un gitano a Guernica.

Guernica, per di più, entra nel racconto come antefatto ; un antefatto importante ma che resta comunque tale, risolvendo il destino della città ferita in poche, scontate battute e avviando una storia d'amore minata dal razzismo, in un contesto che finisce per evadere la stessa guerra di Spagna. Un gitano si trova per caso in quella città, il 26 aprile 1937 . Ovvero si trova nel posto sbagliato al momento sbagliato, anche se " Guernica, quel giorno, non lo sembrava davvero un posto sbagliato ", con " giovani stesi sui prati a prendere il sole e a chiaccherare " e "soldati tranquilli " : "Chi avrebbe mai immaginato che, di lì a poco, la terra si sarebbe sollevata così in alto da raggiungere il cielo. Il gitano sentì il rumore degli aerei, alzò gli occhi e vide il cielo oscurato di apparecchi [...] Quel giorno a Guernica si sperimentava, per la prima volta nella storia, il bombardamento aereo di una città ". Il risultato, tristemente noto, viene poi riassunto, con l'aggiunta di una sentenza, discutibile, che lascia intravedere il destino analogo di altre città spagnole ed europee negli anni bui a venire : « Della città rimase in piedi ben poco. Cittadini e soldati si aggiravano tra le macerie travolti dallo stupore della novità. In guerra è sempre così, l'orrore colpisce e indigna la prima volta, poi diventa routine ». Il punto di vista del gitano, che tende a farsi gli affari suoi anche a bombardamento cominciato, si perde dunque, insieme ai suoi cari, tutti caduti quel giorno, nel proseguio della guerra civile, durante la quale, combattendo per i repubblicani, conosce una bellissima basca. Scoppia l'amore, finisce la guerra e il gitano

47. Aldo Garosci, Gli intellettuali e la guerra di Spagna, Torino, Einaudi, 1959, p. 421. 
progetta una fuga a due quando siamo già, per lo meno, al giugno del 1940, visto che nel racconto si legge : « italiani e tedeschi avevano appena invaso la Francia ». Impaziente, il gitano attende la sua bella a una stazione spagnola ma la vede scendere dal treno in compagnia di un franchista. Credendosi tradito, la uccide e fugge nell'America del Sud. Raggiunto dai fratelli di lei, dai quali apprende la sua innocenza, chiede : "Per cosa morirò, oggi. Per averla uccisa o perché lei voleva fuggire con me? ». Gli si risponde con una domanda che secca qualsiasi speranza - "Che differenza fa ? " - e il gitano muore, diventando racconto, nell'osteria : " la storia di un gitano che un giorno era salito su un treno che andava dalla parte sbagliata, come tutta la sua vita».

Scorrerei ancor più velocemente Camilleri, il cui brevissimo racconto, tutto italiano, è incentrato comicamente su uno scambio di persona in cui la guerra civile spagnola ha una parte relativa. Ma è vero che Andrea Camilleri, preso in toto, meriterebbe forse un discorso più vasto, per la pressoché costante relazione della sua narrativa con la storia, point d'ancrage dei siciliani; e anche, per esempio, a livello di certi innesti biografici (ovviamente più disseminati e parziali rispetto al concentrato ramondiniano a cui sopra si alludeva).

Camilleri è del 1925 e si può dunque permettere, nel suo racconto, un incipit testimoniale nel quale far poi scivolare un attacco narrativo classico : " Al tempo della guerra di Spagna, che io avevo una decina d'anni, al largo del mio paese, Porto Empedocle, passavano spesso navi mercantili sovietiche cariche di viveri e di medicinali [...]. Ogni tanto incappavano in posti di blocco della Marina militare italiana ed erano sottoposte a perquisizioni lungariose per vedere se c'erano armi ammucciate nelle stive. Una notte scatasciò un temporale di mala intenzione $[\ldots]$.

Passiamo dunque a Bruno Arpaia e al suo Ĺangelo della storia, dicendo subito che la parte del romanzo che più ci interessa coincide, pressapoco, con la sua prima metà, con quelle prime centoquaranta pagine alla fine delle quali si assiste a un debole ma significativo passaggio di consegne, sul quale vale la pena di intrattenersi per la particolare struttura del romanzo di cui si è detto. Da un lato, infatti, abbiamo le città spagnole ferite fino alla primavera del 1939 e dall'altro la capitale francese colpita, l'alba del 3 giugno 1940, dalla Luftwaffe, che bombarda Parigi, sintesi urbana altissima di " mille autres fins du monde, au cours des millénaires", secondo un libro strano ma a tratti illuminante come Le matin des magiciens 
$(1960)^{48}$; libro strano che storicamente, e apocalitticamente, mette insieme tanti dati, molte città ferite, diversi incendi, e nel caso specifico di Parigi prosegue evocando il rogo di Berlino ${ }^{49}$. Del resto è pur vero che un amante un po' particolare della capitale francese come Hitler ${ }^{50}$, pensa a Parigi distrutta dal fuoco e forse si fa profeta della distruzione di Berlino, percependo quasi in un dionisiaco annientamento estetico quelle due realtà urbane dove strategie e leggende oscure hanno poi finito per intrecciarsi e dare più o meno buoni risultati romanzeschi : da quelli francesi di Paris brûle-t-il ?, del 1964, di Lapierre e Collins, a quelli italiani de Il rogo di Berlino, del 1995, di Helga Schneider ${ }^{51}$.

Nel romanzo di Arpaia, però, si parla di un altro amante di Parigi, Walter Benjamin, che «non ebbe il tempo di prendere la maschera antigas e scendere giù in strada, perché il bombardameno finì presto, lasciando in aria un odore di carne bruciaticcia, di calcinacci e di polvere da sparo che gli arrivava a folate dalla finestra aperta, mischiato alle sirene delle ambulanze e dei pompieri ${ }^{52} »$. È un punto limite del romanzo, dal quale, per i nostri fini, bisognerebbe partire, per leggere il testo all'indietro e cogliere « il rigor mortis della guerra » che Koestler riesce a fuggire « senza lasciare traccia » ma dal quale Benjamin stenta ad allontanarsi, « immobile» come la città ferita, che non può che attendere il prossimo bombardamento, con " la voce di un profeta, calma [...] di chi si affida alla catastrofe per riaggiustare il mondo "in un baleno" ", fermo, seduto dietro quella "scriva

48. Louis Pauwels e Jacques Bergier Le matin des magiciens. Introduction au réalisme fantastique, Paris, Gallimard, 1960 e Ivi, « Folio », 1972, p. 241-242.

49. Che è poi solo il punto terminale, potremmo dire, di tutte le città tedesche devastate da assedi terrestri e celesti di cui si incomincia a parlare con più fervore in Germania e nel mondo per denunciare, a vari livelli d'indagine, saggistico, storico e letterario, una realtà spesso taciuta dai vincitori del secondo conflitto mondiale : penso, per le tante città tedesche ferite dai bombardamenti, al libro di Jörg Friedrich, Der Brand, del 2003, e, per i rapporti tra bombardamenti, guerra aerea e letteratura, alle conferenze tenute a Zurigo nel 1997 da Sebald e raccolte nel volume di Winfried Georg Sebald, Lufkrieg und Literatur, München/Wien, Carl Hanser Verlag, 1997, tradotto di recente in francese : cfr. Id., De la destruction comme élément de l'histoire naturelle, Arles, Actes Sud, 2004.

50. Cfr. Arno Breker, Hitler : Paris à la sauvette, in «Historia », 1973, 319, p. 106-117

51. Cfr. Dominique Lapierre, Larry Colins, Paris brûle-t-il ?, Paris, Laffont, 1964, e Helga Schneider, Il rogo di Berlino, Milano, Adelphi, 1995, dove sono pagine alte sulla presa della città da parte dei russi che ricordano l'anonima Una donna a Berlino, Milano, Mondadori, 1957 e ora Torino, Einaudi, 2004. Sui russi a Berlino cfr. ancora Erich Kuby, Die Russen in Berlin 1945, Munchen-BernWien, Scherz Verlag, 1965 (trad. it. Torino, Einaudi, 1966), e il più recente Antony Beevor, Berlino 1945, Milano, Rizzoli 2003.

52. Per questa citazione e le seguenti cfr. B. Arpaia, L'angelo della storia cit., p. 148, 145, 138 e $136,78$. 
nia» della Bibliothèque Nationale che diventa la sua "Linea Maginot ». Fra il testamento, firmato, delle Tesi sul concetto di storia e gli infiniti, quasi anonimi Passages, grande libro di citazioni in cui " ogni frase era scritta come se fosse la prima ad affacciarsi al mondo, o l'ultima prima della catastrofe ${ }^{53}$ ", l'opera e Walter Benjamin, indissolubilmente legati, si specchiano insieme, nel mondo delle città ferite, e ne diventano una sorta di doppio : "Il libro si sarebbe presentato come un campo di macerie agli occhi di un superstite che, ferito, si risveglia all'indomani di una battaglia ».

Prima delle settanta pagine à rebours appena percorse, dove il destino di Benjamin e quello di Parigi si legano sempre più, fra 1939 e 1940, emerge l'altro e ben diverso registro della narrazione di Arpaia, affidato al combattente repubblicano Laureano Mahojo, che fa l'amore finanche sotto i bombardamenti di Barcellona, pur incontrando qualche incoveniente di natura tecnica per l'evidente stress di copulare sotto le bombe ; luogo comune che è anche in Guernica di Lucarelli e che, nel contesto della città ferita, offre una variante del binomio 'sesso e morte', traduzione del più celebre e meno volgare 'amore e morte. Si seguono così, dall'inizio del romanzo, in un clima più tragicomico ma sostanzialmente serio, i destini urbani di Gijón, la città del narratore, che "sembrava un girone dell'inferno, illuminata a giorno dagli incendi ${ }^{54}$ "; Bilbao, nel cui cielo, all'improvviso, "venivano cinquanta caccia, settanta trimotori, a martellarci con le mitragliatrici e con le bombe "; la citata Barcellona, dove, finito il bombardamento, si esce dal rifugio, si scherza e si ride con le ragazze, ma constatando che « c'era poco da ridere, là fuori. Non si contavano, i morti e i feriti. Dovunque fumo e polvere, e le sirene delle ambulanze che andavano e venivano. Palazzi interi si erano sbriciolati come se fossero stati fatti di torrone, nelle strade si aprivano voragini e l'acqua che sprizzava dalle tubature avvolgeva in lenti mulinelli i corpi dei povericristi che ci erano rimasti intrappolati ». E poco dopo la stessa Barcellona viene presentata come una " città intontita dai bombardamenti », grazie all'accanirsi degli italiani che "partivano dalla base aerea di Maiorca e scaricavano più bombe che potevano ». Tra l'altro, propri i latini sono i responsabili del

53. Cfr. a questo proposito Susan Sontag, Sotto il segno di Saturno (1978), in Id., Sotto il segno di Saturno, Torino, Einaudi, 1982, p. 89-110 ; in particolare p. 106-108.

54. Per questa citazione e le seguenti cfr. B. Arpaia, L'Angelo della storia, cit. p. 10 e 16, 11, $24,35,36,68,75,177$. E per Port Bou, in un contesto miliziano, cfr. un poco noto antecedente italiano in Vittorio Bodini, La lobbia di Masoliver (1968-1969) in Id., La lobbia di Masoliver, Milano, Scheiwiller, 1980, p. 89-96; in particolare p. 94. 
coito interrotto evocato, che lascia libero il campo ad alcuni luoghi comuni di questa nuova guerra moderna e tecnologica, come quello, già visto nel racconto di Carlotto, dei bombardamenti a tappeto, continui : «Arrivavano a ondate, i bombardieri, più o meno ogni tre ore, colpendo ogni quartiere, ogni obiettivo civile e militare. Mai visto uguale. Era la prima volta nella storia, e io mi ci trovavo in mezzo ". Siamo vicini, insomma, alla caduta di Barcellona, il 26 gennaio del 1939, città che ormai si evoca quasi soltanto in relazione ai "bombardamenti, sempre più forti sempre più spaventosi ». Sembra di sentire - ma con segno mutato - un cinegiornale fascista del gennaio '39 : «Già l'aviazione legionaria, anticipando la marcia liberatrice delle truppe, domina i cieli della Catalogna. Già le aquile d'acciaio sovrastano la città che attende... Sono gli ultimi giorni di Barcellona rossa ». E «Barcellona caduta nelle mani dei franchisti » è evocata infatti nel proseguio del romanzo di Bruno Arpaia ma sconfina già, per quel passaggio di consegne di cui si è detto, nella narrazione dedicata a Walter Benjamin e a Parigi. Anche se punto d'incontro e rendez-vous ultimo dei due diversi destini esistenziali non è una grande città ferita ma un paese, Port Bou : anch'esso, comunque, ferito, con le " macerie della guerra ancora in giro ».

Quando il romanzo di Arpaia appare in Francia, col titolo mutato in Dernière frontière, Lionel Richard, recensendolo, in appendice al dossier del « Magazine littéraire » dell'aprile 2002, dedicato a Walter Benjamin, commenta : " un roman qui n'est qu'une coquille bourrée d'artifice dans un bricolage conventionnel ${ }^{55}$ ". C'è del vero anche se il giudizio è forse troppo severo e parziale, essendo basato, sostanzialmente, sulla parte benjaminiana del romanzo.

La parte migliore del testo di Arpaia - con i limiti che abbiamo visto e che vanno ribaditi - è quella dedicata a Laureano, le cui gesta, eroiche ed erotiche, sono colte, in parallelo, insieme al destino di Benjamin e dell'Europa, quasi come una sorta di controcanto comico-serioso alla tragicità degli anni Trenta e del 1940. Nell'Angelo della storia si gioca troppo, come si è già suggerito, sul bipolarismo combattente-corpo e intellettuale-testa. Verso la fine ${ }^{56}$, Benjamin insiste per consegnare il suo testamento, le tesi citate, Sul concetto di storia, testo di estrema densità speculativa e di inci-

55. « Magazine littéraire », 2002, 408, p. 59-60 ; citazione da p. 59.

56. Per questa citazione e le seguenti cfr. B. Arpaia, L'angelo della storia cit., p. 249, 205, 159-161 (e 175). 
siva laconicità, a un uomo che "non capiva perché quei fogli scritti fitti fitti fossero più importanti di lui [Benjamin] e della sua vita ", a un uomo che nel 1940, a un anno dalla fine della guerra civile, fa il contrabbando per amore e si sveglia, una di quelle mattine in cui incrocia il filosofo, con pensieri di tal fatta : "Si sa, le donne, anche se non lo dicono, non ce n’è una a cui non piaccia essere ripassata la mattina presto, avvolte in quell'odore di dormito, rimbambolate ancora per il sonno, quasi meravigliate ». È davvero il contrasto eccessivo, didascalico, a frenare il ritratto dell'intellettuale tedesco sulle solite coordinate : un 'topo da biblioteca' la cui resistenza, fatta di lettura e scrittura, è talmente iterata e assolutizzata, anche a livello morale, da diventare banale ; un uomo inetto, solitario, chiuso, che però alla fine si apre con il primo che arriva, quasi riconoscendo un alter ego (veramente altro, qu'on se le dise). Chi si emancipa, poi, non è neppure il soldato-contrabbandiere, che da ottuagenario racconta al romanziere tutta la sua vita e tutto quello che sa del filosofo e critico tedesco e che finisce per incarnare un luogo comune : quello della resistenza della vita fondata su ben altri pensieri, come quelli citati poc'anzi. Insomma, un po' di sesso, in guerra, ti salva dalle bombe, da Franco e finanche, in un'altra guerra, dai nazisti e dal governo di Vichy. Certo, il tono d'Arpaia è serio-serioso nei confronti di Benjamin e cerca un vero ritmo storico, storico-biografico, che sposa anche squarci narrativi e battute felici, azzeccate, ma facili e un po' stancamente iterate, come quella giocata intorno a Le Rouge et le Noir di Stendhal : "A rue de l'Odéon, la libreria era chiusa, Adrienne non c'era ancora [...] Adrienne aprì le imposte [...]. "Parto, ho deciso" confessò Benjamin [...] "è proprio sicuro di partire ? Parigi è così grande... È il posto ideale per nascondersi" " [questa che parla è Adrienne Monnier ma sembra Benjamin, che invece dice] «"[...] lei non conosce i tedeschi quanto me" [...] le mani si agitavano attorno a un libro di Stendhal [...]. "Allora" si decise a dire "già che ci siamo, le posso chiedere di regalarmi anche Il rosso e il nero ? Vorrei rileggerlo ma la mia copia è dispersa da anni per l'Europa..." Ventiquatt'ore dopo, i tedeschi attraversavano la Senna $[\ldots]^{57}$ ".

Bello, forse pure esatto, ma davvero facile. Tout se tient proprio dove e quando tout ne se tient pas, nella vita vera, difficile, vagabonda di Benjamin e nell'Europa a pezzi di quegli anni. La ratio di Bruno Arpaia, comunque, regola la contaminazione, sfumandola, ed allontana gli eccessi del

57. Per Adrienne Monnier e Sylvia Beach cfr. il recente e ricco volume di Laure Murat, Passage de l'Odéon. Sylvia Beach, Adrienne Monnier et la vie littéraire à Paris dans l'entre-deuxguerres, Paris, Fayard, 2003. 
romanzesco postmoderno di Tutto il ferro della Torre Eiffel - il titolo allitterativo è la cosa migliore - di Michele Mari, pubblicato da Einaudi nel 2002. Ambizioso, divertente ma davvero fine a se stesso, come qualche recensore, a livello storico davvero competente e creativo, ha potuto suggerire $^{58}$.

Abbiamo sopra accennato, con Arpaia, a Maiorca e ai bombardieri italiani che dalla base aerea dell'isola partono con il loro carico di morte verso le città spagnole in mano ai rossi. Tante le immagini che tornano su questa realtà nel lungo romanzo di Fabrizia Ramondino, Guerra di infanzia e di Spagna, che è, per l'appunto, d'ambientazione maiorchina e che recupera le prime Storie di patio (1983), La signora di Son Batle, I servi, Gli uccelli di Narcis, Il fidanzamento ${ }^{59}$, servendole al lettore leggermente modificate nella Parte prima.

Per le immagini relative alla realtà della guerra, dei bombardamenti, c'è quasi l'imbarazzo della scelta, perché L'isola incantata, come recita il secondo capitolo della Parte prima del romanzo, ospita l'immaginario infantile della piccola protagonista ma anche, subito, la guerra : "Dalle stecche delle persiane filtrava il sole, e il profumo dei mandorli in fiore avvolgeva a folate l'isola, ma in cielo si udiva il rombo degli aerei levatisi in volo a bombardare castelli o caserme ${ }^{60}$ ". L'avversativa è forte ma non apre sulle città ferite. Di fatto, Ramondino seguirà raramente il volo degli aerei fino alla loro infuocata meta urbana. Il punto di vista che li insegue li perde prima, magari in un campo dell'isola, come ne L'uccello della guerra. Del resto, nemmeno le Notizie della guerra - capitolo della Parte quarta vogliono completare il quadro in questo senso, né quelle poste in Appendice e già citate. Quello che più interessa a Fabrizia Ramondino è enunciare una certa contaminazione dell'Eden, del bel parco d'infanzia ${ }^{61}$. In questa

58. Cfr. Alessandro Barbero, La letteratura come feticcio. Benjamin e il nano malefico, in "L'Indice ", 2003, 1, p. 8. Sull'uso che di alcuni grandi critici e personaggi intellettuali del Novecento, fra Walter Benjamin (1892), per l'appunto, Giacomo Debenedetti (1901) e Roberto Bazlen (1902), ha fatto la narrativa degli ultimi vent'anni, da Del Giudice a Arpaia e Mari, ho tenuto una lezione a Firenze il 12 novembre 2003, il cui testo, di prossima pubblicazione, era titolato Un angelo, un dandy e " un nomo a cui piaceva vivere negli interstizi della cultura e della storia".

59. Cfr. F. Ramondino, Storie di patio cit., p. 3-92.

60. Per questa citazione e i seguenti rinvii cfr. F. Ramondino, Guerra di infanzia e di Spagna cit., p. 23, 227-229 (e 197), 305-368 e 415-417.

61. Sull'infanzia e la guerra civile spagnola si vedano, in questa prospettiva, ma senza la mediazione dell'isola incantata, alcune pagine di François-Marie Ribadeau, Le pain et la pierre, Atelier Marcel Jullian, 1979, p. 67-75, relative al cap. IV, Guerre civile dans le village. 
prospettiva - con tonalità forti e crude che la avvicinano a un'altra scrittrice partenopea, un po' più giovane, Elena Ferrante $(1943)^{62}$ - un dito ficcato nell'ano alla bambina da un amante della capocameriera, " un chiodo di carne " - che si traduce nella parola « tortura » e poi « tormenti », come quelli delle "vergini martiri cristiane e delle suore di Barcellona ${ }^{63}$ " - sembra valere una bomba, un bombardamento e anticipare la Storia del bambino di Guernica, la violenza, la tortura, il tormento che il nin ha dovuto subire, nella realtà storica e non fuori di essa, e quindi anche nelle città ferite dalla guerra e non nell' isola che non c'è. Non è un caso, tra l'altro, che nella Storia del bambino di Guernica vi sia un antefatto di natura sessuale, con un giovane quindicenne che si farà frate perché, innamoratosi di una bella vicina di casa, ne cercherà i favori ma troverà solo un "seno nudo [...] tutto piagato, divorato dal cancro ", che verrà interpretato, fra sacro e profano, come una sorta di rivelazione, di « Nuestra Senora de la Teta ». Insomma, il corpo o l'anima dell'uomo è il luogo vero, fisico, della ferita. Tanto che sulla città distrutta dal bombardamento il 26 aprile 1937 l'autrice propone il secco racconto del frate ormai âgé, ma cercando, significativamente, un legame con ciò che della guerra tutti sanno e vivono a Maiorca, compresi i bambini. In questo senso, chi scrive sembra quasi chiudere, in un immaginario infantile e isolato, il racconto. Quel racconto che poi invece si apre su una specie di apocalisse tesa ad evadere il solito luogo comune della nuova guerra moderna e tecnologica, nonostante il facile, risaputo attacco che la introduce : «Ed ecco, all'improvviso, fu l'inferno ! Il cielo rombò. A quel rumore mi si gelò il sangue; lo conoscevo bene quel rumore. Tutti lo conoscono bene a Maiorca. Ma non erano uno, due aerei, nemmeno una squadriglia di cinque. Erano centinaia, oscuravano il cielo, lucevano, sputavano fuoco e fiamme. Non sapevo se fuggire o gettarmi per terra, o ancora accovacciarmi coprendomi il volto con le mani. "Ma no, - mi dissi - Voglio vedere in faccia Dio !" E rimasi in piedi con gli occhi al cielo ». E quel Dio - che è poi, se vogliamo, il diavolo di Lucarelli e del genere letterario che frequenta - lascia Guernica in questo stato : « La città era un ammasso di rovine, di sangue, di lamenti ; ebbene, era proprio simile al seno che mi aveva mostrato la mia donna. Tutta putredine e piaghe ». Eccolo il point d'ancrage di Fabrizia Ramondino, che del resto si intuiva ben prima, come si è detto.

62. Penso qui, soprattutto, a L'amore molesto (1992).

63. Per questa citazione e le seguenti cfr. F. Ramondino, Guerra di infanzia e di Spagna, cit., p. 63, 202-203, 206-207 e 207. 
La traduzione simbolica che la scrittrice partenopea mette in bocca a quel saltimbanco di frate, a un personaggio marginale alla Soler, ha il sapore amaro di una sentenza ed è strutturalmente ineccepibile. La città ferita è davvero, in queste pagine, il corpo dell'uomo ferito, torturato dal male dell'esistenza, della Storia. Non ci sono mediazioni e astrazioni intellettuali e quelle religiose e metafisiche accrescono, con un po' di ironia, la parabola drammatica del fatto storico, amplificandola a partire da un universo infantile che non solo non ne è immune ma che ne diventa, suo malgrado, protagonista.

Non a caso l'evocazione delle macerie di Guernica porta al ritrovamento del nin, che è come se fosse stato torturato dal bombardamento : " per tre giorni girai fra le macerie a soccorrere i feriti e i morenti. Trovai il nin in una piazzetta sotto un albero divelto. Pareva dormire, era invece in coma, gravemente ferito ; e quasi a volersi prendere gioco di lui, gli era finita nel cavo del braccio una palla... ». Dopo le cure apportategli in un convento, che è posto significativamente "fuori città ", il bambino guarisce ma non parla : "Non parla, sapete, non tanto per la paura che s'è preso, ma per l'orrore che, se parlasse, sarebbe costretto a raccontare al mondo». 